The continuance of the investigations of the Division of Radiophysics into the practicability of increasing rainfall by seeding clouds with silver iodide by a further large-scale field trial is in progress in the Northern Tablelands region of New South Wales. Development continued of a method for obtaining bearings from existing Distance Measuring Equipment Beams and also research into the purification of semiconductor materials and growth of mono crystals, the transport of charges in semiconductors and the development of junction photo devices. An all-sky camera was installed in October 1957 and photographs of the whole night sky have been taken regularly at five-minute intervals since that time, recording any auroræ that may occur, and in conjunction with cameras at other stations, enabling positions and height of auroræ to be deduced. In solar physics the association between a class of radio bursts conventionally known as type II and optical features in the chromosphere has been investigated, while the main observational programme of the 15-metre wave-length "Cross" aerial was directed towards completing a survey of a belt of the sky, $10^{\circ}$ wide, around the galactic equator. The crossed-grating interferometer is producing each day a detailed radio picture of the Sun, and observations of solar radio disturbances continued throughout the year with the Dapto radio spectrograph, which records the Sun's spectrum in the range of wavelengths between about 1.5 and $7.5 \mathrm{~m}$. The Mathematical Instruments Section completed the construction of the transistorized digital differential analyser and the techniques are being applied to the development of a small general-purpose computer.

\title{
DELAYED HYPERSENSITIVITY IN IMMUNOLOGY
}

T HE mechanism of the delayed form of hypersensitivity, originally and still exemplified by Koch's tuberculin reaction, has proved much more difficult of analysis than that of the immediate reaction about the main immunological features of which much is now known. In the symposium on "Delayed Hypersensitivity" held by the British Society for Immunology in London on May 8, the allergic phenomena associated with reactions of this type provided the central theme for discussion.

Little progress towards the understanding of the mechanism of the tuberculin reaction can be expected, as pointed out by $\mathrm{S}$. V. Boyden in opening the Symposium, until the nature of the specific change in the tissues responsible for the hypersensitivity is recognized and can be detected and measured in vitro. The injection of tuberculoproteins, when in soluble form, leads to the production of specific antibodies in the blood but not to the appearance of delayed hypersensitivity. Even when these proteins are adsorked on carbon granules or red cell stromata to provide them with a particulate vehicle, their injection almost invariably results in the development of Arthus-type hypersensitivity. It seems that it is only when these antigens enter the tissues as an integral part of the bacillus, and consequently pass through some intracellular experience in phagocytes, that the animal will respond to a subsequent skin test with a typical delayed tuberculin reaction.

In part, the characteristic delay in the development of the tuberculin reaction might be attributable, as $d$. Pepys has observed, to the period of several hours needed for the full fixation of the provocative agent to the tissue cells. The simultaneous injection of any agent, such as histamine or hyaluronidase which can accelerate the loss of the tuberculin from the site of inoculation, or of adrenalin which can ensure its retention in the area, consequently much affects the intensity of the ensuing reaction. It follows, therefore, that any constituent present in the tuberculin preparation used that might evoke even a relatively inconspicuous immediate reaction could, by so doing, lead to the dispersal of the factor which was the cause of the delayed reaction and so mask any later manifestations of delayed hypersensitivity. On the other hand, the introduction of the tubereulin in a lipid vehicle prolongs the local retention of the tuberculin, thereby enhancing its potency and revealing in man the presence of degrees of hypersensitivity too low to be demonstrable by intracutaneous tests with large doses of tuberculin.

The discovery by Landsteiner and Chase that in guinea pigs specific delayed hypersensitivity can be transferred by an inoculum of leucocytes when one of serum is ineffective has been further analysed for human beings by H. S. Lawrence. He sought, by making extracts from such cells after their lysis, to identify the 'transfer factor' concerned. This he found to be a stable agent capable of resisting exposure to deoxyribonuclease, ribonuclease and trypsin. With Pappenheimer, he found that it may be liberated from the sensitizing leucocytes by incubation alone or by contact with tuberculoproteins; the latter procedure caused the cells themselves to lose their distinctive property of sensitizing a recipient. Delayed hypersensitivity to coccidioidin is similarly transferable with extracts of sensitizing leucocytes and the specific systemic reactivity so conferred may persist for more than a year.

In seeking some biological meaning for delayed hypersensitivity reactions, Lawrence proposed an extension of Burnet and Fenner's 'self-marker' concept to postulate that interaction between host cells and phagocytozed microbes may produce slightly altered versions of the individual's cellular components by forming intimate (self plux $x$ ) complexes. The latter, recognized as foreign by the host, may provoke a cellular immune response (transfer factor) directed against the complex. The cellular immune response takes effect against the host's own tissues in the form of a local homograft reaction wherever and whenever his cells are in appropriate combination with the antigen $(x)$ which has induced the alteration. The effector mechanism (transfer factor) is uncovered following transfer to recipients and in the presence of the test antigen $(x)$ it is postulated that it evokes a train of events similar to that called forth by the intact microbe in the cells of the donor.

N. A. Mitchison further followed up the possible resemblances between delayed hypersensitivity and the homograft reaction by pointing out that in both, the immunological responses appeared to be attributable to the participation of cell-bound antibody. A graft of tissue from one animal to another of the 
same species provokes the production of both humoral and cell-bound antibody; but the former generally does not destroy the graft. Transplantation immunity thus possesses an important feature in common with delayed hypersensitivity. Further similarities arise from the routes of immunization used - the intravenous injection of cells provokes only a poor response in the rabbit-as well as from the tempo of the full transplantation immunity reaction which develops before the production of humoral antibody reaches its maximum. More significantly, the cellular infiltration of homografts resembles the granuloma induced by tubercle bacillary wax. The nature of the cell first stimulated by the antigen may determine the type of the response, so that a single antigen may, in different circumstances, elicit either a humoral or a cell-bound antibody. Alternatively, tissue cells may be supposed to possess isoantigens of different kinds each responsible for one kind of antibody.

The possibility that a single molecular species of antigen can provoke simultaneously both delayed and immediate sensitivity to different determinant groups on it was discussed by P. G. H. Gell and B. Benacerraf. In a study of various types of immunological reaction to proteins conjugated with such active haptenes as picryl chloride, they have demonstrated a dissociation between immediate and delayed skin reactivity to the same antigen. The absence of recognizable antibodies to the protein carriers used in these conjugates, at a time when their intradermal injection proved capable of exciting a delayed skin reaction, confirms the view that reactions of this type do not depend on conventional antibodies in the circulation : at this time, antibodies were present specific to the haptenic group. Under other conditions, delayed sensitivity to the haptenic group was also demonstrable. They questioned the view that the state of delayed hypersensitivity can be regarded as an early, perhaps immature, stage of immunity. Rather, they felt that it should be considered as a distinctive response to certain quali. tatively different, possibly less dominant, groups on the antigenic molecule.

With the recognition of the close participation of leucocytes in the transference of specific delayed hypersensitivity, J. L. Gowans's account of the lifehistory of lymphocytes acquires particular relevance. Experiments on rats have shown that the output of these cells from the thoracic duct is sufficient to replace all the lymphocytes in the blood many times daily; the production of new small lymphoeytes is much lower and their survival time much longer than was formerly supposed. These cells, moreover, appear to circulate freely through the tissue spaces and in this extravascular transit they may be the effector cells in immunological reactions of the delayed type.

The features of the immediate and delayed 'tuberculin-type' reactions to trichophytin in guinea pigs that can be evoked either after an infection or an inoculation with the killed mycelium were described by C. N. Cruickshank, M. D. Trotter and M. R. Wood. They found that these responses were associated with a transferable passive cutaneous anaphylaxis, but that they could occur in the absence of any detectable precipitating antibodies. Chemical fractionation of the mycelium showed that the antigenic material was mainly a polysaccharide containing equal proportions of glucose and mannose. Finally, R. M. Gordon and M. Lavoipierre, in discussing immediate and delayed reactions to insect bites, pointed out that in certain instances the late reactions ordinarily attributed to the saliva of the vector may be confused with that caused by some parasite introduced into the tissues at the time.

\section{THE ELECTRIC ARC IN WELDING}

$\mathrm{F}^{\circ}$ OLLOWING the practice of the previous two years, a third "Joining of Metals" Conference was held at the University of Birmingham on June 25 under the chairmanship of Prof. E. C. Rollason, head of the Department of Industrial Metallurgy. The subject on this occasion was "The Electric Arc in Welding"; five papers were presented, and the conference was attended by about one hundred representatives of industry, the research associations and the universities. Prof. Rollason explained that the purpose of these conferences was to further the development and teaching of the basic processes underlying the practice of metal joining, and pointed out that, in arc welding in particular, much less effort had been directed towards fundamentals than to the empirical development of modern are welding processes. $\mathrm{He}_{\theta}$ then suggested that there were three ways in which the arc interacted with the metal which were of significance in welding. First, there was heat transfer from the arc which was responsible for the formation of the weld pool; secondly, the chemico-metallurgical reactions taking place between the high-temperature gases in the arc atmosphere and the weld metal; and thirdly, the transfer of metal droplets across the arc which can take place against gravity and for which no satisfactory mechanism had yet been advanced.

Mr. D. R. Milner, of the Department of Industrial Metallurgy, then surveyed the present state of knowledge of those aspects of arc physics which were pertinent to these problems. Throughout the main body of the arc electrical energy is utilized to heat the gas to such a temperature that it becomes thermally ionized and is thus able to provide the electrons and ions necessary to carry the required current. At the anode the electron stream, and at the cathode the positive ion stream, provide the source of energy for melting the metal. For low-current ares the anode processes are reasonably well established, but less is known of conditions at the cathode. However, for high-current welding systems little information is available in either case. Heat and mass transfer from the arc column to the electrodes, which determ. ines the reactions occurring between the arc atmosphere and the weld motal and controls the rate at which they take place, is dependent upon the energy dissipated, the gas properties and the mode of heat transfer. Of particular interest in this respect are plasma-jets, which Maecker has demonstrated exist wherever there is a constriction in the arc, such as a 